\title{
Identification and Manipulation of Memory Engram Cells
}

\author{
Xu Liu, ${ }^{1,2,3}$ Steve Ramirez, ${ }^{1}$ Roger L. Redondo, ${ }^{1,2}$ and Susumu Tonegawa ${ }^{1,2}$ \\ ${ }^{1}$ RIKEN-MIT Center for Neural Circuit Genetics at the Picower Institute for Learning \\ and Memory, Department of Biology and Department of Brain and Cognitive Sciences, \\ Massachusetts Institute of Technology, Cambridge, Massachusetts 02139 \\ ${ }^{2}$ Howard Hughes Medical Institute, Massachusetts Institute of Technology, Cambridge, \\ Massachusetts 02139 \\ Correspondence: tonegawa@mit.edu
}

\begin{abstract}
How memories are formed and stored in the brain remains a fascinating question in neuroscience. Here we discuss the memory engram theory, our recent attempt to identify and manipulate memory engram cells in the brain with optogenetics, and how these methods are used to address questions such as how false memory is formed and how the valence of a memory can be changed in the brain.
\end{abstract}

How and where memory is stored in the brain network is one of the fundamental questions in brain and cognitive sciences. At the onset of the 20th century, a German biologist Richard Semon proposed the engram theory of memory (Semon 1923), but the theory was nearly completely ignored by his contemporary and subsequent brain researchers, until Daniel Schactor, James Eich, and Endel Tulving revived the theory in the late 1970s (Schacter et al. 1978). Semon's memory engram theory was built on two fundamental postulates termed the "Law of Engraphy" and the "Law of Ecphory" for memory storage and memory retrieval, respectively. The Law of Engraphy posits: "All simultaneous excitations (derived from experience) ... with in our organisms form a connected simultaneous complex of excitations which, as such, acts engraphically, that is to say leaves behind it a connected, and to that extent, unified engram-complex" (Semon 1923). The Law of Ecphory on the other hand posits: "The partial return of an energetic situation which has fixed itself engraphically acts in an ecphoric sense upon a simultaneous engram-complex" (Semon 1923).

Semon's conceptualizations of the memory process were novel for his time and were remarkably predictive of the contemporary concepts of memory storage and retrieval. For instance, Semon's memory retrieval process contained the concept of "pattern completion," which was advanced years later (Marr 1970; Nakazawa et al. 2003; Leutgeb et al. 2004). However, Semon did not elaborate the biological basis of the "simultaneous excitations" nor "a connected, unified engram-complex." This is not surprising considering that his theory was put forward nearly a century before the development of molecular, cellular, and genetic biology and sophisticated imaging and electrophysiological technologies for the analysis of the nervous system.

Incorporating the current knowledge about neurons, synaptic connections, and neuronal circuits, Semon's Engram Theory of Memory can be rephrased as follows:
When a subject undergoes or encounters an episode, a set of selected stimuli from the experience or episode activate populations of neurons to induce enduring physical and/or chemical changes (engrams) in them and their connections, each contributing to the storage of memory. Subsequently, when a part of the original stimuli returns, these cells (engram cells) are reactivated to evoke the recall of the specific memory.

A half-century after Semon's book was published, Karl Lashley pioneered a systemic hunt for engram cells in the rodent brain by introducing lesions of varying sizes into different areas of the cerebral cortex, attempting to find an engram for a maze task. However, Lashley found that memory was impaired in many of these lesioned animals, and the severity of the impairments was proportional to the sizes of the lesions. On the basis of these findings, Lashley concluded that the engrams for maze-resolving memory are spread throughout the cerebral cortex with no obvious localization (Mass Action Principle) (Lashley 1950). However, soon after Lashley's study, Wilder Penfield and Theodor Rasmussen obtained the first evidence suggesting that the engrams of episodic memories are stored in the medial temporal lobes (MTLs) (Penfield and Rasmussen 1950). This chance finding was supported several years later by William Scoville and Brenda Milner, who discovered that a patient H.M., who lost a large portion of his MTLs as a result of surgery, had severe anterograde amnesia for episodes as well as a graded retrograde amnesia. These studies were consistent with the notion that episodic memories are stored in the MTLs.

As to the nature of memory engrams - enduring physical and chemical changes induced by learning-the guiding hypothesis has been Donald Hebb's theory, which posits that neurons encoding memory stimuli undergo enduring strengthening of some of their synapses through their coactivation with presynaptic cells: neurons that "fire together wire together" (Hebb 1949). Starting with Tim Bliss and Terje Lomo's discovery of long-term

\footnotetext{
${ }^{3}$ Present address: Department of Neurobiology, Northwestern University, Evanston, Illinois 60208.

Copyright (C) 2014 Cold Spring Harbor Laboratory Press; all rights reserved; doi: 10.1101/sqb.2014.79.024901

Cold Spring Harbor Symposia on Quantitative Biology, Volume LXXIX
} 
potentiation (Bliss and Lomo 1973), which supports Hebb's hypothesis, a large number of studies have been directed to the characterization of LTP and other facets of synaptic plasticity and their potential role in learning and memory. However, none of these studies could link these activity-dependent alterations of synapses and neurons directly to engram cells, which are activated by specific learning and whose reactivation by the specific recall cues elicited behavioral responses.

In this review, we shall outline our recent attempt to identify memory engram cells and to manipulate them by optogenetics to investigate several thus far unresolved issues associated with episodic memory.

\section{OPTOGENETIC ACTIVATION OF MEMORY ENGRAM CELLS}

Previous studies have linked selected neuronal populations with particular memory events by correlational evidence (Reijmers et al. 2007) and loss-of-function evidence (Han et al. 2009; Zhou et al. 2009), but a critical piece of evidence was largely missing. The most direct evidence of engram cells should come from gain-of-function experiments, where a population of neurons that are considered responsible for a particular memory are selectively labeled and activated artificially to mimic their natural activity. If such manipulation causes the recall of that memory, then this provides evidence that the selected population of neurons is sufficient for the memory, and thus argues the selected neuronal population is the neuronal basis for the engram of this particular memory (Martin and Morris 2002). However, this type of gain-offunction experiment is technically challenging, as one has to be able to correctly isolate the neurons involved in one particular memory from their seemingly indistinguishable neighbors and activate them with proper spatial and temporal precision. Recent advances in technology such as optogenetics (Fenno et al. 2011; Goshen 2014) enabled such experiments.

To achieve this goal, we combined activity-dependent, drug-regulatable expression system with optogenetics (Liu et al. 2012). We used a transgenic mouse model where the artificial tetracycline transactivator (tTA), which can be blocked by doxycycline (Dox), is driven by the promoter of immediate early gene (IEG) $c$-fos (Reijmers et al. 2007). The activity dependency of $c$-fos promoter poses a natural spatial constrain on the identities of the neurons that can be labeled, reflecting the normal biological selection process of the brain during memory formation, whereas the Dox-dependency of the system poses an artificial temporal constrain as to when these neurons can be labeled, which can be controlled by the experimenters. With these two constraints, the downstream effector of tTA can express selectively in neurons that are active during a particular behavior episode, only if the animals are off Dox diet. Using this system, we expressed channelrhodopsin-2 (ChR2) delivered by a viral vector AAV-TRE-ChR2-EYFP targeting the dentate gyrus (DG) of the hippocampus and implanted optical fibers right above the infected areas (Fig. 1A). These animals were habituated in one context A with light stimulation while on Dox, and then taken off Dox and fear conditioned in context B, where DG neurons active during the formation of this context-fear association memory were labeled by ChR2 (Fig. 1B). After which they were put back on Dox diet to stop further labeling and tested again in context A by light stimulation of the labeled neurons (Fig. 1C).

Although light had no effect on the test subjects in context A before training, these animals showed reversible, light-dependent freezing in context A after training (Fig. 1D), indicating light-induced recall of fear memory associated with fear conditioning, which happened in context B. Because these animals did not freeze in con-

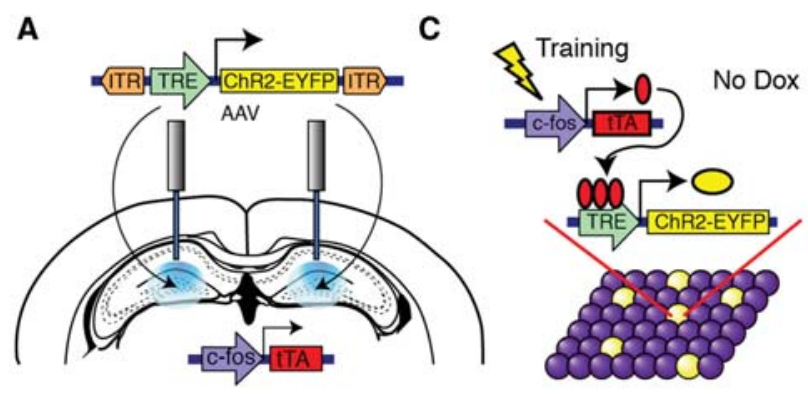

B

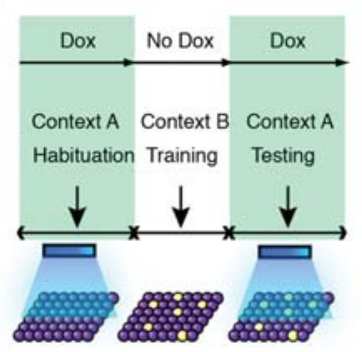

D

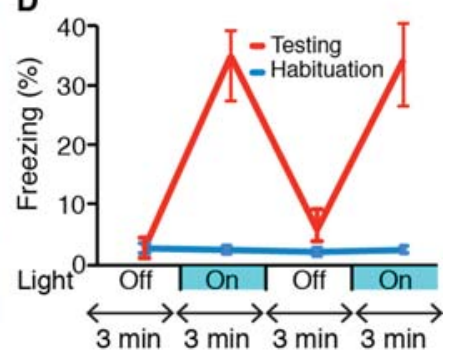

Figure 1. Optogenetic activation of memory engram cells induced memory recall. (A) Basic composition of the system. Virus expressing TRE-ChR2 and optic fibers are targeted bilaterally into the dentate gyrus (DG) of transgenic mouse line expressing c-fos-tTA. $(B)$ In the absence of Dox, DG neurons that are active during the formation of a memory are labeled with ChR2. (C) Behavior schedules. Animals were habituated to context A with light stimulation while on Dox, trained in context $\mathrm{B}$ while off Dox, and texted again in context A with light stimulation while on Dox. (D) Although light had no effect during pretraining habituation sessions, the animals showed light-dependent freezing behavior posttraining, indicating the light-induced recall of a fear memory. 
text $\mathrm{A}$ in the absence of light posttraining, this ruled out the possibility that the freezing was due to generalization between context $\mathrm{A}$ and $\mathrm{B}$. Control animals underwent similar treatments except did not receive footshock in context B did not show light-induced freezing after training, although a similar number of DG neurons were labeled after exposure to context B with or without shock (Liu et al. 2012). This indicated that simply activating a population of DG neurons not associated with a contextual fear memory by light stimulation was not the cause of freezing. Another control group underwent the same treatment as the experimental group including the footshock in B, but expressed only EYFP instead of ChR2 and also failed to show light-induced freezing after training, indicating that potential sensitization of light after fear conditioning also could not account for the behavior. These two controls reflected the requirement of both the presence of a fear memory and light activation of neurons associated with that memory for the observed light-induced freezing, and thus supported the idea that in the experimental group, activation of neurons associated with a previous fear memory by light indeed caused the ectopic recall of that memory in an otherwise neutral context (Liu et al. 2012). Taken together, these experiments provided the gain-of-function evidence for the memory engram cells, suggesting these cells are sufficient for the memory.

The artificial recall of the memory was also faithful to some extent, as it showed contextual specificity. Experiments have shown that two statistically independent (Liu et al. 2012) or even distinct (Deng et al. 2013) populations of DG neurons are active in two different contexts. We examined the cross talk between neuronal ensembles representing different contexts by testing if artificial activation of neurons representing one context could induce the recall of memory associated with another context. If the neurons active in a neutral context A were labeled while the animals were off Dox, followed by fear conditioned in a different context B while on Dox, the animals did not display freezing behavior upon light stimulation in a third context (Liu et al. 2012). This results showed that light activation of a neuronal population associated with a neutral context will not elicit the recall of another fear-conditioned context, as long as the neuronal representation of these two contexts are orthogonal, thus supporting the context specificity of the light-induced fear memory recall. This observation is also consistent with the proposed patter separation function of the DG (Leutgeb et al. 2004). This study showed that the behavior expression of a memory could be controlled from the neuronal ensemble level and opened possibilities for further memory manipulations using the engram cell method, as we will discuss below.

\section{GENERATING FALSE MEMORIES BASED ON MEMORY ENGRAM CELLS}

The experimental realization of reactivating discrete memories in the rodent brain (Liu et al. 2012) enabled subsequent studies to test long-standing hypotheses about the malleability of memory with unprecedented spatialtemporal resolution (Ramirez et al. 2013). These notions began in the early 1930s when the British psychologist Frederic Bartlett recited slightly inconsistent fables to people from several cultural backgrounds, most famously The War of the Ghosts (Bartlett 1932). While recalling the fable, many subjects unintentionally modified the contents of the story into a logical narrative that contained new elements that fit within their cultural milieu. Bartlett discovered that streams of recalled contextual information could act as a modifiable scaffold onto which information is added or distorted. Indeed, memories are not immutable video records of the experienced past that are projected onto a mental theater; they are mnemonic rivers that ebb and flow and thereby reconstruct the neuronal riverbeds that structurally support various streams of information. Bartlett had discovered the labile nature of memory.

Since Bartlett, the process of memory "updating" has been experimentally shown in both humans and rodents. Rats given electroconvulsive shocks shortly after recalling an aversive memory subsequently display profound amnesia for the original event (Misanin et al. 1968). This process of memory updating, later termed "reconsolidation," was rediscovered in 2000 and shown to be dependent on protein synthesis in the lateral amygdala (Nader et al. 2000) or hippocampus depending on the type of memory recalled (Debiec et al. 2002). Examples abound in humans that highlight the dramatic instances in which distorted memories of crime scenes, childhood events, and traumatic experiences - often recalled under interrogation in the court of law or during psychotherapy sessions - disrupt both individual well-being and modern jurisprudence (Loftus 2003; Schacter and Loftus 2013).

Of course, although the rich repertoire of human false memories is difficult to fully model in animals, a starting point is to take a Pavlovian approach and deconstruct the learning process into conditioned stimuli (CSs) and unconditioned stimuli (USs) associations. A series of recent studies have successfully showed the proof of principle of artificially linking CSs and USs to form novel associative memories. For instance, Johansen et al. (2010) showed that optically activated lateral amygdala (LA) cells were sufficient to substitute as a US during tone (CS) presentations and, upon subsequent tone presentations, animals displayed fear behavior despite the CS and US having never been naturally, or exogenously, presented. Another study showed that an activated population of pyriform cortex neurons, when paired with rewards or shocks, could drive the associated appetitive or aversive behavioral output upon stimulation of the same neurons (Choi et al. 2011). Moreover, pairing footshocks with optogenetically reactivated secondary auditory cortex and medial geniculate nucleus (MGN) inputs to the LA was also sufficient to form an associative fear memory to the optically activated terminals (Kwon et al. 2014). A more recent study elegantly showed that optically inducing long-term potentiation (LTP) or long-term depression (LTD) from MGN terminals into the LA was 
sufficient to promote or inhibit a previously formed memory, thus engineering the inactivation and reactivation of a specific memory and causally linking its expression to these types of synaptic plasticity (Nabavi et al. 2014).

Finally, a context-specific artificial memory was recently achieved using the "engram-labeling" technology (Ramirez et al. 2013). Animals were first taken off Dox to open a window for activity-dependent labeling and exposed to a neutral environment (context A). Thus, DG cells active during the exploration of context $A$ became ChR2-positive (Fig. 2A). While back on Doxand thus the window for activity-dependent labeling was closed-animals were then exposed to context C. The following day, fear conditioning occurred in context B with or without light stimulation of DG cells. In this experiment, even though the animals had a memory for both context $\mathrm{A}$ and $\mathrm{C}$, only the neuronal populations activated by the former was ChR2-positive and presumably reactivated during fear conditioning in context $\mathrm{B}$. We hypothesized that, if DG cells previously active during context A exploration defined an active neural population sufficient for memory recall, then optogenetic activation of these cells during fear conditioning in context B should form an artificial association between the light-induced context A memory and aversive information (e.g., footshocks). To test this possibility, animals were first placed back in context $\mathrm{C}\left(\mathrm{C}^{\prime}\right)$ and showed low basal levels of freezing (Fig. 2A). However, when placed back in context A $\left(\mathrm{A}^{\prime}\right)$, only the animals in which DG cells were reactivated displayed robust freezing behavior, indicating that a false memory for context A had been artificially formed. Moreover, when placed in another neutral environment (context D), control animals showed basal levels of freezing during light-off and light-on epochs, whereas the experimental group in which an artificial memory had been created displayed robust freezing behavior only during light-on epochs (Fig. 2B). In other words, the expression of the false memory was behaviorally similar to the lightinduced expression of a natural fear memory as previously reported (Liu et al. 2012).

Together, these data suggest that optical reactivation of hippocampal dentate gyrus cells that were previously active during context exploration is sufficient to act as an artificial, context-specific CS during fear conditioning. This thereby forms an artificial CS-US association, or a putative false memory, because the artificially constructed memory never had its contiguous experiences naturally linked (Ramirez et al. 2013). These results are consistent with the temporal context model (TCM) in humans, which posits that contextual memory reactivation can be linked to novel information that is presented at the time of reactivation (Gershman et al. 2013; St Jacques and Schacter 2013). Notably, the formation of false memories in humans often occurs as a result of recombining mnemonic elements of discrete experiences into a new, reconstructed memory that is not a veridical representation of the past. These memories are not de novo and require preexisting memories as a scaffold onto which distinct experiences can be incorporated to update the memory itself (Tse et al. 2007; Gershman et al. 2013). Similarly, in all rodent studies to date, any artificial memories generated were not de novo constructions; rather, they are results of artificially linking either a preexisting memory or concurrent learning processes with events of high valence.

\section{CHANGING MEMORY VALENCE THROUGH MEMORY ENGRAM CELLS}

After demonstrating the possibility to assign negative valence to an original neutral DG engram (Ramirez et al.
A
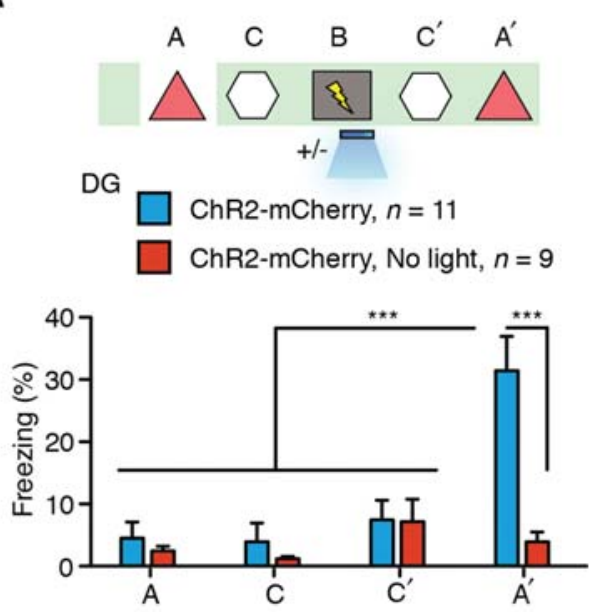

B
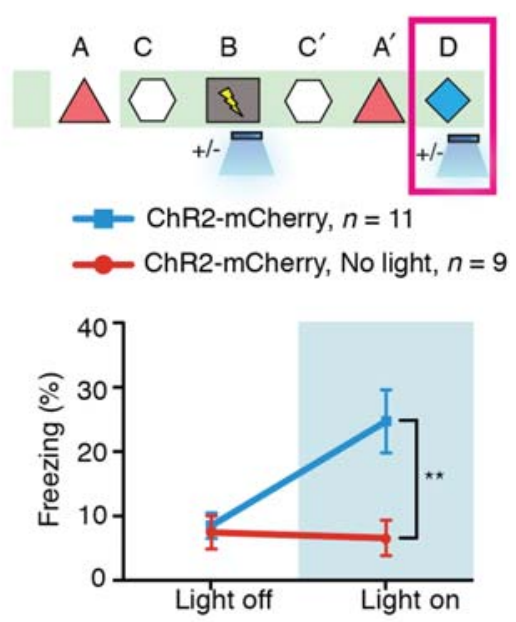

Figure 2. Creation of a false memory. $(A)$ (Top) c-fos-tTA mice injected with AAV 9 -TRE-ChR2-mCherry in the DG were taken off Dox and exposed to context A to label correspondingly activate DG cells with ChR2-mCherry and then put back on Dox and exposed to context $\mathrm{C}$ the following day. A control group injected with $\mathrm{AAV}_{9}-\mathrm{TRE}-\mathrm{ChR} 2-\mathrm{mCherry}$ did not receive light stimulation during fear conditioning (ChR2-mCherry, no light). (Bottom) animals' freezing levels in context A and C before and after fear conditioning with or without light revealed that a false memory had been formed only in the group in which DG stimulation occurred during fear conditioning in context $\mathrm{B} ;\left({ }^{* * *} P<0.001\right)$. $(B)$ Animals that underwent the behavioral protocol shown in the top panel were exposed to a novel context $\mathrm{D}$, and the freezing levels were examined both in the absence and presence of light stimulation $\left({ }^{* *} P=0.007\right)$. 
2013), we set out to identify the nodes of the circuit capable of associating valence to contextual representations. If the DG processes contextual information, the main hub for emotional learning in the brain is the amygdala, a group of nuclei deep in the temporal lobe that when lesioned impairs emotion but spares memory in tasks without emotional components (Zola-Morgan et al. 1991; Anderson and Phelps 2001). What are the contributions of the DG and the amygdala to the memory engram? In Redondo et al.'s (2014) study, neuronal ensembles that up-regulated c-fos at the time of memory encoding were manipulated in the DG or in the basolateral amygdala (BLA). Active avoidance responses were detected when mice reduced the time spent in a side of a maze where laser stimulation was delivered (OptoPA test). Active appetitive responses were identified by an increase in the time spent inside the zone targeted with laser (OptoPP test). First, we showed that both DG and BLA engrams are capable of driving an aversive response (day 5; Fig. 3A,B). Then, after pairing the optogenetic reactivation of the DG engram while mice experience a positive emotion (i.e., female contact, day 7 in Fig. 3A), the circuit changed so that subsequent activation of the DG ensemble drove an appetitive behavioral response on day 9 (Fig. 3A,B) and no longer produced an aversive response (Fig. 3C). After the induction protocol on day 7 , the BLA engram failed to produce an appetitive response on day 9 (Fig. 3B) and retained the ability to drive an aversive response upon its activation (Fig. 3C). A symmetrical result was obtained when DG ensembles originally linked to an appetitive memory were optogenetically reactivated during fear learning: The output of the DG engram then drove an aversive response (Redondo et al. 2014).

To understand how the functional connectivity between the DG and the BLA had changed, the DG and the BLA engram were simultaneously labeled in a group of mice during memory encoding (Fig. 3D). On day 3, mice were divided into three groups: one experienced a US of opposite valence to that encoded on day 1 without laser stimulation $\left(\mathrm{US}^{+}, \mathrm{Light}^{-}\right.$); another received an induction protocol pairing the presentation of a US of op-
A

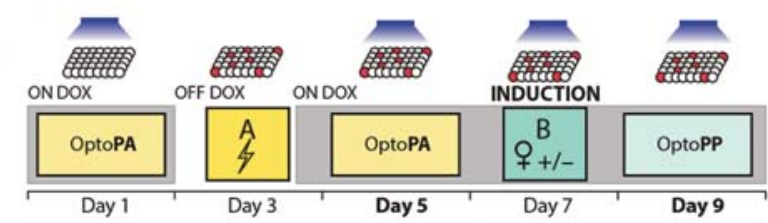

B

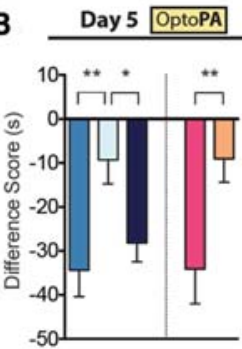

DG-ChR2

BLA-ChR2

$\mathbf{F}$

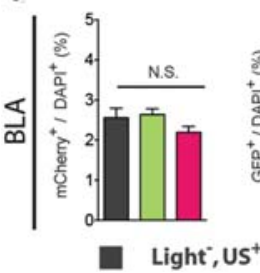

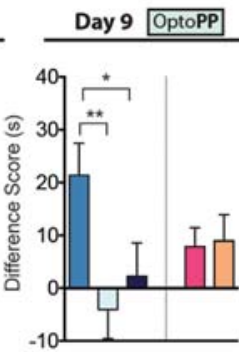

DG-mCherry-only BLA-mCherry-only

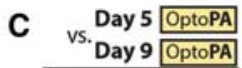

DG-ChR2-NoUS-Day?

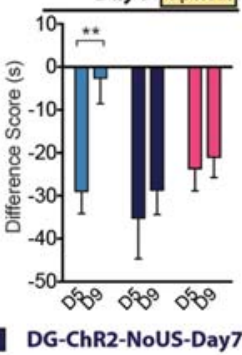

D

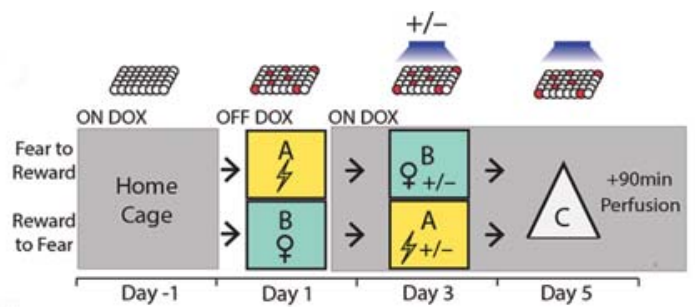

E
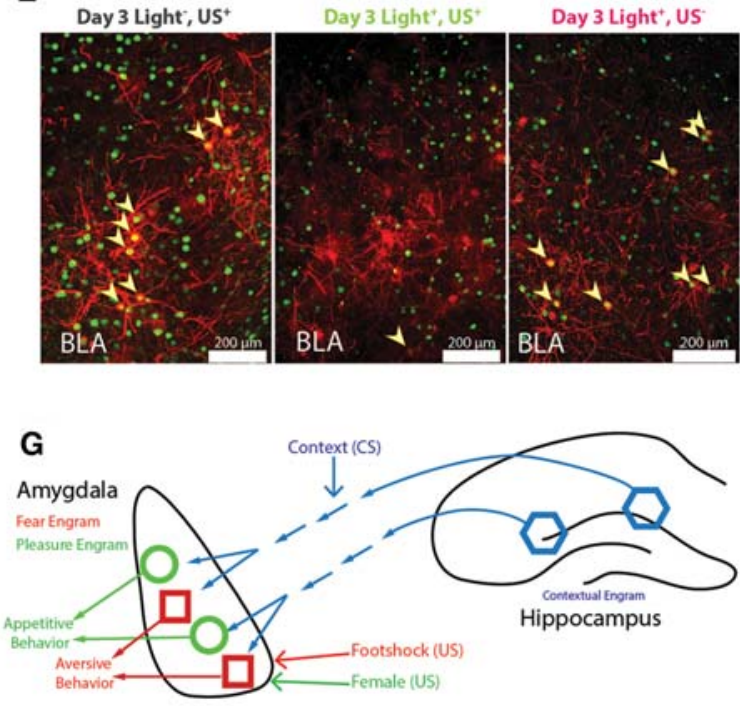

Figure 3. Reversal of the valence associated with the hippocampal memory engram. $(A)$ Daily protocol with labeling of fear memory engram (day 3) and later its reactivation during a pleasurable experience (day 7). (B) Only in the DG engram but not in the BLA engram, the aversive response driven on Day 5 (reduction in time spent in the area where the memory engram is reactivated) is reversed into an appetitive response on day 9. (C) Only in the DG engram but not in the BLA engram, the aversive response on day 5 is eliminated on day 9. $(D)$ Daily protocol depicting the labeling of an engram (day 1 ) followed by induction (day 3 ) and ending in the reactivation of the DG engram and brain extraction (day 5). (E) Representative images of the BLA showing engram neurons labeled on day 1 (red) and neurons reactivated on day 5 (green). All groups show high overlap except the group that underwent the valence reversal protocol. $(F)$ Bar graphs quantifying similar levels of labeling and activation in the BLA across groups. However, only the group that underwent induction shows a reduced reactivation score that approximates to chance levels. $(G)$ Summary graph depicting the potential of contextual information from the hippocampus to associate with amygdala neurons specialized in driving appetitive or aversive responses. 
posite value to that encoded with optogenetic stimulation (Light ${ }^{+}, \mathrm{US}^{+}$), and the last group received optogenetic stimulation without US delivery $\left(\mathrm{Light}^{+}, \mathrm{US}^{-}\right)$. On day 5 all animals had the DG ensembles reactivated optogenetically and $90 \mathrm{~min}$ later the brains were collected and processed for immunohistochemistry. Green fluorescence revealed those neurons that had recently up-regulated cfos, whereas red fluorescence identified the neurons expressing ChR2-mCherry labeled on day 1 (Fig. 3E). The laser stimulation delivered to the DG was equally effective across groups at reactivating the DG engram (data not shown). Also in the BLA, the three experimental groups had the same amount of cells labeled $\left(\mathrm{mCherry}^{+} /\right.$ $\left.\mathrm{DAPI}^{+}\right)$and the same levels of c-fos activation $\left(\mathrm{GFP}^{+} /\right.$ $\mathrm{DAPI}^{+}$) (Fig. 3F). However, although the control groups $\left(\mathrm{US}^{+}, \mathrm{Light}^{-}\right.$and $\mathrm{Light}^{+}, \mathrm{US}^{-}$) showed high levels of reactivation, the animals that experienced the induction protocol on day 3 had a decreased level of reactivation very close to the levels expected by chance (Fig. 3E,F). Two main conclusions can be drawn from these data: First, activating the DG ensemble reactivates the BLA neuronal population colabeled during memory encoding; and second, the induction protocol changes the connectivity between the DG and the BLA in such a way that even though the DG drives a similar number of neurons in the BLA, the identity of the activated BLA population has changed and no longer corresponds to those neurons that were active during memory encoding. That is, after reversing the valence associated with a DG ensemble, the output from the DG drove a different population of amygdala neurons.

The emerging picture of the circuit for memory valence depicts a series of neutral components of the engram (DG) free to associate with either positive or negative valences coded by nodes downstream in the circuit (BLA) (Fig. 3G). The development of new technologies capable of altering the connectivity between the nodes in the circuits of memory valence opens up the possibility of circumventing classical approaches to the treatment of emotional psychopathologies (i.e., posttraumatic stress disorder [PTSD], depression).

\section{CONCLUSION}

By combining activity-dependent gene expression system and optogenetics, we have established a system where we can identify and manipulate neurons that are active during the formation of a memory. Using this system, several important discoveries were made related to the mechanisms of memory. First, activation of these cells induced the recall of the associated memory, indicating that these cells are sufficient for the memory (Liu et al. 2012). Together with other studies with observational and loss-of-function evidence (Reijmers et al. 2007; Han et al. 2009; Zhou et al. 2009), this gain-offunction experiment pinpointed these cells as the cellular basis of memory engram. Second, artificial activation of these cells made the associated memory labile and capable of incorporating new information to form a new memory (Ramirez et al. 2013). This could be a potential mechanism whereby false memories are formed. Third, pairing the activation of a memory of certain valence while experiencing an event of opposite valence can reverse the valence originally associated with the memory. This alteration of memory valence is possibility due to changes of functional connectivity between the hippocampus and the amygdala (Redondo et al. 2014).

What we discussed here are just some examples of memory-related questions that can be answered by this new engram cell-based technology. There are ample equally exciting yet unexplored topics waiting to be addressed. For example, what plasticity changes are happening inside these cells harboring memory engrams? What are other memory engram pathways inside the brain? Can we use this technology to tackle disease models for mental disorders, such as depression and anxiety? With the fast evolving new technology, we have reason to believe that the memory engram cell-related studies will continue to bring deeper insights and exciting new discoveries in the years to come.

\section{ACKNOWLEDGMENTS}

We thank P. Pang, C. Puryear, A. Govindarajan, P. Lin, J. Suh, M. Pignatelli, T.J. Ryan, J. Kim, and A. Arons for help with the experiments and all the members of the Tonegawa laboratory for their support. This work was supported by RIKEN Brain Science Institute and Howard Hughes Medical Institute to S.T.

\section{REFERENCES}

Anderson AK, Phelps EA. 2001. Lesions of the human amygdala impair enhanced perception of emotionally salient events. Nature 411: 305-309.

Bartlett FC. 1932. Remembering: A study in experimental and social psychology. Cambridge University Press, Cambridge.

Bliss TV, Lomo T. 1973. Long-lasting potentiation of synaptic transmission in the dentate area of the anaesthetized rabbit following stimulation of the perforant path. J Physiol 232: $331-356$.

Choi GB, Stettler DD, Kallman BR, Bhaskar ST, Fleischmann A, Axel R. 2011. Driving opposing behaviors with ensembles of piriform neurons. Cell 146: 1004-1015.

Debiec J, LeDoux JE, Nader K. 2002. Cellular and systems reconsolidation in the hippocampus. Neuron 36: 527-538.

Deng W, Mayford M, Gage FH. 2013. Selection of distinct populations of dentate granule cells in response to inputs as a mechanism for pattern separation in mice. Elife 2: e00312.

Fenno L, Yizhar O, Deisseroth K. 2011. The development and application of optogenetics. Annu Rev Neurosci 34: 389-412.

Gershman SJ, Schapiro AC, Hupbach A, Norman KA. 2013. Neural context reinstatement predicts memory misattribution. $J$ Neurosci 33: 8590-8595.

Goshen I. 2014. The optogenetic revolution in memory research. Trends Neurosci 37: 511-522.

Han JH, Kushner SA, Yiu AP, Hsiang HL, Buch T, Waisman A, Bontempi B, Neve RL, Frankland PW, Josselyn SA. 2009. Selective erasure of a fear memory. Science 323: 1492-1496.

Hebb D. 1949. The organization of behavior. Wiley \& Sons, New York.

Johansen JP, Hamanaka H, Monfils MH, Behnia R, Deisseroth K, Blair HT, LeDoux JE. 2010. Optical activation of lateral 
amygdala pyramidal cells instructs associative fear learning. Proc Natl Acad Sci 107: 12692-12697.

Kwon JT, Nakajima R, Kim HS, Jeong Y, Augustine GJ, Han JH. 2014. Optogenetic activation of presynaptic inputs in lateral amygdala forms associative fear memory. Learn Mem 21: $627-633$.

Lashley K. 1950. In search of the engram. Symp Soc Exp Biol 4: 454-482.

Leutgeb S, Leutgeb JK, Treves A, Moser MB, Moser EI. 2004. Distinct ensemble codes in hippocampal areas CA3 and CA1. Science 305: 1295-1298.

Liu X, Ramirez S, Pang PT, Puryear CB, Govindarajan A, Deisseroth K, Tonegawa S. 2012. Optogenetic stimulation of a hippocampal engram activates fear memory recall. Nature 484: $381-385$.

Loftus E. 2003. Our changeable memories: Legal and practical implications. Nat Rev Neurosci 4: 2-5.

Marr D. 1970. A theory for cerebral neocortex. Proc R Soc Lond B Biol Sci 176: $161-234$

Martin SJ, Morris RG. 2002. New life in an old idea: The synaptic plasticity and memory hypothesis revisited. Hippocampus 12: 609-636.

Misanin JR, Miller RR, Lewis DJ. 1968. Retrograde amnesia produced by electroconvulsive shock after reactivation of a consolidated memory trace. Science 160: $554-555$.

Nabavi S, Fox R, Proulx C, Lin J. 2014. Engineering a memory with LTD and LTP. Nature 511: 348-352.

Nader K, Schafe GE, LeDoux JE. 2000. The labile nature of consolidation theory. Nat Rev Neurosci 1: 216-219.

Nakazawa K, Sun LD, Quirk MC, Rondi-Reig L, Wilson MA, Tonegawa S. 2003. Hippocampal CA3 NMDA receptors are crucial for memory acquisition of one-time experience. $\mathrm{Neu}$ ron 38: $305-315$.
Penfield W, Rasmussen T. 1950. The cerebral cortex of man: A clinical study of localization of function. Macmillan, New York.

Ramirez S, Liu X, Lin PA, Suh J, Pignatelli M, Redondo RL, Ryan TJ, Tonegawa S. 2013. Creating a false memory in the hippocampus. Science 341: 387-391.

Redondo RL, Kim J, Arons AL, Ramirez S, Liu X, Tonegawa S. 2014. Bidirectional switch of the valence associated with a hippocampal contextual memory engram. Nature 513: $426-$ 430.

Reijmers LG, Perkins BL, Matsuo N, Mayford M. 2007. Localization of a stable neural correlate of associative memory. Science 317: 1230-1233.

Schacter DL, Loftus EF. 2013. Memory and law: What can cognitive neuroscience contribute? Nat Neurosci 16: 119123.

Schacter DL, Eich JE, Tulvlng E. 1978. Richard Semon's theory of memory. J Verbal Learn Verbal Behav 17: 721-743.

Semon R. 1923. Mnemic philosophy. Allen \& Unwin, Australia.

St Jacques PL, Schacter DL. 2013. Modifying memory: Selectively enhancing and updating personal memories for a museum tour by reactivating them. Psychol Sci 24: 537-543.

Tse D, Langston RF, Kakeyama M, Bethus I, Spooner PA, Wood ER, Witter MP, Morris RGM. 2007. Schemas and memory consolidation. Science 316: 76-82.

Zhou Y, Won J, Karlsson MG, Zhou M, Rogerson T, Balaji J, Neve R, Poirazi P, Silva AJ. 2009. CREB regulates excitability and the allocation of memory to subsets of neurons in the amygdala. Nat Neurosci 12: 1438-1443.

Zola-Morgan S, Squire LR, Alvarez-Royo P, Clower RP. 1991. Independence of memory functions and emotional behavior: Separate contributions of the hippocampal formation and the amygdala. Hippocampus 1: 207-220. 


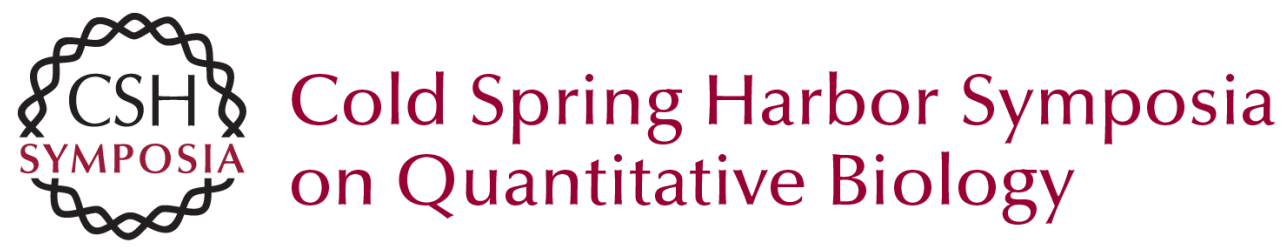

\section{Identification and Manipulation of Memory Engram Cells}

Xu Liu, Steve Ramirez, Roger L. Redondo, et al.

Cold Spring Harb Symp Quant Biol 2014 79: 59-65 originally published online January 30, 2015 Access the most recent version at doi:10.1101/sqb.2014.79.024901

References This article cites 30 articles, 9 of which can be accessed free at: http://symposium.cshlp.org/content/79/59.full.html\#ref-list-1

\section{License}

Email Alerting Receive free email alerts when new articles cite this article - sign up in Service the box at the top right corner of the article or click here. 